\title{
Pertuzumab/Trastuzumab/Paclitaxel Followed by FEC Regimen
}

National Cancer Institute

\section{Source}

National Cancer Institute. Pertuzumab/Trastuzumab/Paclitaxel Followed by FEC

Regimen. NCI Thesaurus. Code C138046.

A chemotherapy regimen consisting of pertuzumab, trastuzumab and paclitaxel, followed by fluorouracil, epirubicin and intravenous cyclophosphamide, used as a neoadjuvant or adjuvant treatment for HER-2/neu-positive breast cancer. 